\title{
A New Method Based on Laplace Transform and Its Application to Stability of Pipe Conveying Fluid
}

\author{
H. B. Wen, ${ }^{1,2}$ Y. R. Yang, ${ }^{2}$ P. Li, ${ }^{2}$ Y. D. Li, ${ }^{1,3}$ and Y. Huang ${ }^{2,4}$ \\ ${ }^{1}$ School of Mechanical Engineering, Sichuan University of Science and Engineering, Zigong 643000, China \\ ${ }^{2}$ School of Mechanics and Engineering, Southwest Jiaotong University, Chengdu 610031, China \\ ${ }^{3}$ Sichuan Province University Key Laboratory of Bridge Non-Destruction Detecting and Engineering Computing, Zigong 643000, China \\ ${ }^{4}$ School of Mechatronic Engineering, Southwest Petroleum University, Chengdu 610500, China
}

Correspondence should be addressed to H. B. Wen; sichuanwhb@suse.edu.cn

Received 31 October 2016; Accepted 26 December 2016; Published 24 January 2017

Academic Editor: Lorenzo Dozio

Copyright (c) 2017 H. B. Wen et al. This is an open access article distributed under the Creative Commons Attribution License, which permits unrestricted use, distribution, and reproduction in any medium, provided the original work is properly cited.

\begin{abstract}
A new differential transformation method is developed in this paper and is applied for free vibration problem of pipes conveying fluid. The natural frequencies, critical flow velocities, and vibration mode functions of such pipes with several typical boundary conditions are obtained and compared with the results predicted by Galerkin method and finite element method (FEM) and with other results archived. The results show that the present method is of high precision and can serve as an analytical method for the vibration of pipes conveying fluid.
\end{abstract}

\section{Introduction}

The dynamics of pipes conveying fluid receives considerable attention always because it contains abundant mechanical phenomena. The equations of motion of the fluid-pipe system are high-order partial differential equations, so the analysis is difficult. Many analytical methods have been proposed to study this dynamic model since Ashley and Haviland [1] began to study the dynamics of straight tubes conveying fluid. These methods include the finite element method (FEM), analytical method, multiple scales method, Galerkin method, transfer matrix method, differential quadrature method, and generalized differential quadrature rule.

Gregory and Paidoussis [2] studied unstable oscillation of tubular cantilevers conveying fluid by an analytical method and Bubnov-Galerkin method. Both parameter and combination resonances for a continuously flexible pipe were studied by Paidoussis and Sundararajan [3], where Bolotin's method and a numerical Floquet analysis were presented. Hannoyer and Paidoussis [4] studied the dynamics and stability of cylindrical tubular beams conveying fluid and simultaneously subjected to axial external flow, where the solutions were obtained by means of a method similar to
Galerkin method, with eigenfunctions approximated by Fourier series. Misra et al. [5, 6] have studied the dynamics and stability of the cantilevered pipes conveying fluid by finite element method; the conclusions were the same as those of Gregory and Paidoussis [2]. Olson and Jamison [7] introduced a general-purpose finite element program to simulate the dynamics of elastic pipes conveying fluid with different boundary conditions. Kuiper and Metrikine [8] employed a $D$-decomposition method for stability analysis of a riser conveying oil from seabed to a floating platform. Wang and $\mathrm{Ni}$ [9] established the nonlinear vibrations of a cantilevered pipe conveying fluid with motion-limiting nonlinear constraints by extending the differential quadrature method (DQM). Utilizing the differential quadrature method again, Qian et al. [10] analyzed the instability of the pinned-pinned pipe conveying fluid under thermal loads. Tornabene et al. [11] extracted the critical flow velocity of straight pipes conveying fluids for various fluid densities by the generalized differential quadrature (GDQ) method. $\mathrm{Ni}$ et al. [12] analyzed the free vibration problem of pipes conveying fluid with several typical boundary conditions by a relatively new semianalytical method called differential transformation method (DTM). Sinir and Demir [13] used 
the method of multiple scales to investigate the vibrations of highly tensioned pipe subject to external force. Quite recently, Li et al. [14] have developed the steady-state Green's functions for forced vibrations of Timoshenko beam with damping effects. Subsequently, Li and Yang [15] employed the same method to study the forced vibration of pipes conveying fluid. Three examples of the pipe conveying fluid were analyzed, the results of which show the validity of the Green function method.

In present paper, a new differential transformation method is developed to analyze the free vibration of pipes conveying fluid. Starting from the basic equations, the variable separation technique and Laplace transforms are employed to derive vibration modes of the pipe conveying fluid, where all the constants involved are determined by the boundary conditions. The vibration mode is a closed and explicit form. Furthermore, a linear system with four equations is obtained by the application of boundary conditions. For the free vibration problem of pipes conveying fluid, the determinant of coefficient matrix of the equations is zero. So the natural frequency equations are derived, which is an implicit form in terms of $\omega$. The natural frequency equation is solved by a computer program. In general, the frequency of the fluid-pipe system will be complex and the dynamic system will be stable or unstable according to the real part or the imaginary part of the frequency being positive or negative.

\section{The Equation of Small Motions}

The equation of small motion of pipes conveying fluid is [16]

$$
\begin{gathered}
E I \frac{\partial^{4} w}{\partial x^{4}}+M U^{2} \frac{\partial^{2} w}{\partial x^{2}}+2 M U \frac{\partial^{2} w}{\partial x \partial t}+(M+m) \frac{\partial^{2} w}{\partial t^{2}} \\
+\mu \frac{\partial w}{\partial t}=0,
\end{gathered}
$$

where $E I$ is flexural rigidity; $U$ is the fluid flow velocity; $M$ and $m$ are the mass-per-unit length of fluid and the pipe, respectively; $\mu$ is the structure damping effect; $w(x, t)$ is the transverse deflection of the pipe; $x$ is the coordinate along the center line of the pipe; and $l$ is the pipe length.

For the sake of illustration, the following dimensionless quantities are introduced:

$$
\begin{aligned}
\eta & =\frac{w}{l}, \\
\xi & =\frac{x}{l}, \\
\beta & =\frac{M}{M+m}, \\
\tau & =\left(\frac{E I}{M+m}\right)^{1 / 2} \frac{t}{l^{2}}, \\
c & =\frac{\mu l^{2}}{\sqrt{E I}}, \\
\bar{u} & =\left(\frac{M}{E I}\right)^{1 / 2} l U .
\end{aligned}
$$

Substitution of these terms into equation (1) yields the dimensionless governing equation of motion

$$
\frac{\partial^{4} \eta}{\partial \xi^{4}}+\bar{u}^{2} \frac{\partial^{2} \eta}{\partial \xi^{2}}+2 \bar{u} \beta^{1 / 2} \frac{\partial^{2} \eta}{\partial \xi \partial \tau}+\frac{\partial^{2} \eta}{\partial \tau^{2}}+c \frac{\partial \eta}{\partial \tau}=0 .
$$

The corresponding dimensionless boundary conditions are as follows:

(i) At a pinned end

$$
\eta= \begin{cases}\frac{\partial^{2} \eta}{\partial \xi^{2}}=0 & \text { at } \xi=0 \\ \frac{\partial^{2} \eta}{\partial \xi^{2}}=0 & \text { at } \xi=1 .\end{cases}
$$

(ii) At a clamped end

$$
\eta= \begin{cases}\frac{\partial \eta}{\partial \xi}=0 & \text { at } \xi=0 \\ \frac{\partial \eta}{\partial \xi}=0 & \text { at } \xi=1 .\end{cases}
$$

(iii) At a free end

$$
\frac{\partial^{2} \eta}{\partial \xi^{2}}= \begin{cases}\frac{\partial^{3} \eta}{\partial \xi^{3}}=0 & \text { at } \xi=0 \\ \frac{\partial^{3} \eta}{\partial \xi^{3}}=0 & \text { at } \xi=1\end{cases}
$$

\section{Vibration Mode Equations and Frequency Equations of the Pipe Conveying Fluid}

Let us consider the motions of the pipe for which the dimensionless lateral deflection is given by

$$
\eta(\xi, \tau)=\operatorname{Re}[W(\xi) \exp (i \omega \tau)]
$$

where $\omega$ is a dimensionless circular frequency defined by

$$
\omega=\left(\frac{M+m}{E I}\right)^{1 / 2} \Omega l^{2}
$$

$\Omega$ being the circular frequency of motion. In general, $\omega$ will be a complex number and the system will be stable or unstable depending on the imaginary component of $\omega, \operatorname{Im}(\omega)$, being positive or negative; in the case of neutral stability, $\omega$ is wholly real and $W(\xi)$ is the mode function.

Substituting equation (7) into equation (3) yields

$$
\frac{d^{4} W}{d \xi^{4}}+a_{1} \frac{d^{2} W}{d \xi^{2}}+a_{2} \frac{d W}{d \xi}+a_{3} W=0,
$$

where

$$
\begin{aligned}
& a_{1}=\bar{u}^{2}, \\
& a_{2}=i 2 \beta^{1 / 2} \bar{u} \omega, \\
& a_{3}=i c \omega-\omega^{2} .
\end{aligned}
$$


To derive the mode function of the pipe conveying fluid, the Laplace transform method with respect to the variable $\xi$ is applied in (9):

$$
\begin{gathered}
\widehat{W}(s)=\frac{1}{s^{4}+a_{1} s^{2}+a_{2} s+a_{3}}\left[\left(s^{3}+a_{1} s+a_{2}\right) W(0)\right. \\
\left.+\left(s^{2}+a_{1}\right) W^{\prime}(0)+s W^{\prime \prime}(0)+W^{\prime \prime \prime}(0)\right],
\end{gathered}
$$

where the parameter $s$ in the transformed domain is a complex variable; $W(0), W^{\prime}(0), W^{\prime \prime}(0)$, and $W^{\prime \prime \prime}(0)$ are constants which can be determined by the boundary conditions of the pipes. that

To obtain the inverse transform of $\widehat{W}(s)$, let us assume

$$
s^{4}+a_{1} s^{2}+a_{2} s+a_{3}=\prod_{j=1}^{4}\left(s-s_{j}\right) .
$$

Thus, the following results can be obtained by the method in $[14,17]$ :

$$
\begin{aligned}
L^{-1} & {\left[\frac{\left(s^{3}+a_{1} s+a_{2}\right)}{\left(s-s_{1}\right)\left(s-s_{2}\right)\left(s-s_{3}\right)\left(s-s_{4}\right)}\right] } \\
= & A_{1}(\xi)\left(s_{1}{ }^{3}+a_{1} s_{1}+a_{2}\right) \\
& +A_{2}(\xi)\left(s_{2}{ }^{3}+a_{1} s_{2}+a_{2}\right) \\
& +A_{3} \xi\left(s_{3}{ }^{3}+a_{1} s_{3}+a_{2}\right) \\
& +A_{4}(\xi)\left(s_{4}{ }^{3}+a_{1} s_{4}+a_{2}\right), \\
L^{-1} & {\left[\frac{\left(s^{2}+a_{1}\right)}{\left(s-s_{1}\right)\left(s-s_{2}\right)\left(s-s_{3}\right)\left(s-s_{4}\right)}\right] } \\
= & A_{1}(\xi)\left(s_{1}{ }^{2}+a_{1}\right)+A_{2}(\xi)\left(s_{2}{ }^{2}+a_{1}\right) \\
& +A_{3}(\xi)\left(s_{3}{ }^{2}+a_{1}\right)+A_{4}(\xi)\left(s_{4}{ }^{2}+a_{1}\right), \\
L^{-1} & {\left[\frac{s}{\left(s-s_{1}\right)\left(s-s_{2}\right)\left(s-s_{3}\right)\left(s-s_{4}\right)}\right] } \\
= & A_{1}(\xi) s_{1}+A_{2}(\xi) s_{2}+A_{3}(\xi) s_{3}+A_{4}(\xi) s_{4}, \\
L^{-1}[ & \left.\frac{1}{\left(s-s_{1}\right)\left(s-s_{2}\right)\left(s-s_{3}\right)\left(s-s_{4}\right)}\right] \\
= & A_{1}(\xi)+A_{2}(\xi)+A_{3}(\xi)+A_{4}(\xi),
\end{aligned}
$$

where $A_{j}(\xi)(j=1,2,3,4)$ are functions specified by

$$
\begin{aligned}
& A_{1}(\xi)=\frac{\exp \left(s_{1} \xi\right)}{\left(s_{1}-s_{2}\right)\left(s_{1}-s_{3}\right)\left(s_{1}-s_{4}\right)}, \\
& A_{2}(\xi)=\frac{\exp \left(s_{2} \xi\right)}{\left(s_{2}-s_{1}\right)\left(s_{2}-s_{3}\right)\left(s_{2}-s_{4}\right)}, \\
& A_{3}(\xi)=\frac{\exp \left(s_{3} \xi\right)}{\left(s_{3}-s_{1}\right)\left(s_{3}-s_{2}\right)\left(s_{1}-s_{4}\right)}, \\
& A_{4}(\xi)=\frac{\exp \left(s_{4} \xi\right)}{\left(s_{4}-s_{1}\right)\left(s_{4}-s_{2}\right)\left(s_{4}-s_{3}\right)} .
\end{aligned}
$$

The inverse transform of (11), which is the mode function of the pipe conveying fluid, can be obtained as follows:

$$
\begin{aligned}
W(\xi)= & L^{-1}\left(\frac{s^{3}+a_{1} s+a_{2}}{s^{4}+a_{1} s^{2}+a_{2} s+a_{3}}\right) W(0) \\
& +L^{-1}\left(\frac{s^{2}+a_{1}}{s^{4}+a_{1} s^{2}+a_{2} s+a_{3}}\right) W^{\prime}(0) \\
& +L^{-1}\left(\frac{s}{s^{4}+a_{1} s^{2}+a_{2} s+a_{3}}\right) W^{\prime \prime}(0) \\
& +L^{-1}\left(\frac{1}{s^{4}+a_{1} s^{2}+a_{2} s+a_{3}}\right) W^{\prime \prime \prime}(0) .
\end{aligned}
$$

Inserting (13a)-(13d) into (15) yields

$$
\begin{aligned}
W(\xi)= & \phi_{1}(\xi) W(0)+\phi_{2}(\xi) W^{\prime}(0)+\phi_{3}(\xi) W^{\prime \prime}(0) \\
& +\phi_{4}(\xi) W^{\prime \prime \prime}(0),
\end{aligned}
$$

where $\Phi_{j}(\xi)(j=1,2,3,4)$ are defined by

$$
\begin{aligned}
& \phi_{1}(\xi)=\sum_{i=1}^{4} A_{i}(\xi)\left(s_{i}^{3}+a_{1} s_{i}+a_{2}\right) \\
& \phi_{2}(\xi)=\sum_{i=1}^{4} A_{i}(\xi)\left(s_{i}^{2}+a_{1}\right) \\
& \phi_{3}(\xi)=\sum_{i=1}^{4} A_{i}(\xi) s_{i} \\
& \phi_{4}(\xi)=\sum_{i=1}^{4} A_{i}(\xi)
\end{aligned}
$$

If the right of equation (3) is the Dirac delta function, the Green function of equation (3) will be derived by the same method as above. Green's function has high precision and computational efficiency in the forced vibration analysis of beam $[14,15]$.

To derive the frequency equation of the pipe and to determine the constants $W(0), W^{\prime}(0), W^{\prime \prime}(0)$, and $W^{\prime \prime \prime}(0)$, 
it is necessary to calculate the various order derivatives of $W(\xi)$. From (16), we can obtain

$$
\begin{aligned}
W(\xi)= & \phi_{1}(\xi) W(0)+\phi_{2}(\xi) W^{\prime}(0) \\
& +\phi_{3}(\xi) W^{\prime \prime}(0)+\phi_{4}(\xi) W^{\prime \prime \prime}(0), \\
W^{\prime}(\xi)= & \phi_{1}^{\prime}(\xi) W(0)+\phi_{2}^{\prime}(\xi) W^{\prime}(0) \\
& +\phi_{3}^{\prime}(\xi) W^{\prime \prime}(0)+\phi_{4}^{\prime}(\xi) W^{\prime \prime \prime}(0), \\
W^{\prime \prime}(\xi)= & \phi_{1}^{\prime \prime}(\xi) W(0)+\phi_{2}^{\prime \prime}(\xi) W^{\prime}(0) \\
& +\phi_{3}^{\prime \prime}(\xi) W^{\prime \prime}(0)+\phi_{4}^{\prime \prime}(\xi) W^{\prime \prime \prime}(0), \\
W^{\prime \prime \prime}(\xi)= & \phi_{1}^{\prime \prime \prime}(\xi) W(0)+\phi_{2}^{\prime \prime \prime}(\xi) W^{\prime}(0) \\
& +\phi_{3}^{\prime \prime \prime}(\xi) W^{\prime \prime}(0)+\phi_{4}^{\prime \prime \prime}(\xi) W^{\prime \prime \prime}(0),
\end{aligned}
$$

where the various order derivatives of $\phi_{i}(\xi)(i=1,2,3,4)$ are as follows:

$$
\begin{aligned}
& \phi_{1}^{(k)}(\xi)=\sum_{i=1}^{4} s_{i}{ }^{k} A_{i}(\xi)\left(s_{i}{ }^{3}+a_{1} s_{i}+a_{2}\right) \\
& \phi_{2}^{(k)}(\xi)=\sum_{i=1}^{4} s_{i}{ }^{k} A_{i}(\xi)\left(s_{i}{ }^{2}+a_{1}\right) \\
& \phi_{3}^{(k)}(\xi)=\sum_{i=1}^{4} s_{i}{ }^{k} A_{i}(\xi) s_{i} \\
& \phi_{4}^{(k)}(\xi)=\sum_{i=1}^{4} s_{i}{ }^{k} A_{i}(\xi) .
\end{aligned}
$$

From (18a) to (18d), the intrinsic relations between the physical quantities at the boundary $\xi=0$ and $\xi=1$ can be obtained

$$
\begin{aligned}
& {\left[\begin{array}{llll}
\phi_{1}(1) & \phi_{2}(1) & \phi_{3}(1) & \phi_{4}(1) \\
\phi_{1}^{\prime}(1) & \phi_{2}^{\prime}(1) & \phi_{3}^{\prime}(1) & \phi_{4}^{\prime}(1) \\
\phi_{1}^{\prime \prime}(1) & \phi_{2}^{\prime \prime}(1) & \phi_{3}^{\prime \prime}(1) & \phi_{4}^{\prime \prime}(1) \\
\phi_{1}^{\prime \prime \prime}(1) & \phi_{2}^{\prime \prime \prime}(1) & \phi_{3}^{\prime \prime \prime}(1) & \phi_{4}^{\prime \prime \prime}(1)
\end{array}\right]\left[\begin{array}{c}
W(0) \\
W^{\prime}(0) \\
W^{\prime \prime}(0) \\
W^{\prime \prime \prime}(0)
\end{array}\right]} \\
& =\left[\begin{array}{c}
W(1) \\
W^{\prime}(1) \\
W^{\prime \prime}(1) \\
W^{\prime \prime \prime}(1)
\end{array}\right] .
\end{aligned}
$$

From (4) to (7), we can determine $W(\xi), W^{\prime}(\xi), W^{\prime \prime}(\xi)$, and $W^{\prime \prime \prime}(\xi)$ at $\xi=0$ and $\xi=1$ as follows:

(i) At a pinned end

$$
\begin{array}{r}
W(0)=0, \\
W(1)=0, \\
W^{\prime \prime}(0)=0, \\
W^{\prime \prime}(1)=0 .
\end{array}
$$

(ii) At a clamped end

$$
\begin{gathered}
W(0)=0, \\
W(1)=0, \\
W^{\prime}(0)=0, \\
W^{\prime}(1)=0 .
\end{gathered}
$$

(iii) At a free end

$$
\begin{gathered}
W^{\prime \prime}(0)=0, \\
W^{\prime \prime}(1)=0, \\
W^{\prime \prime \prime}(0)=0, \\
W^{\prime \prime \prime}(1)=0 .
\end{gathered}
$$

For simplicity, the boundary conditions as (21) are considered. Inserting (21) into (20) yields

$$
\left[\begin{array}{ll}
\phi_{2}(1) & \phi_{4}(1) \\
\phi_{2}^{\prime \prime}(1) & \phi_{4}^{\prime \prime}(1)
\end{array}\right]\left[\begin{array}{c}
W^{\prime}(0) \\
W^{\prime \prime \prime}(0)
\end{array}\right]=\left[\begin{array}{l}
0 \\
0
\end{array}\right] .
$$

In order that a nontrivial solution of these equations exists, the determinant of the coefficient matrix of (24) must be zero. Thus, we can obtain the frequency equation of a pinnedpinned pipe conveying fluid as follows:

$$
\phi_{2}(1) \phi_{4}^{\prime \prime}(1)-\phi_{2}^{\prime \prime}(1) \phi_{4}(1)=0 .
$$

Similarly, the frequency equations of a clamped-clamped pipe conveying fluid and a cantilevered pipe conveying fluid can be obtained as follows:

$$
\begin{aligned}
\phi_{3}(1) \phi_{4}^{\prime}(1)-\phi_{3}^{\prime}(1) \phi_{4}(1) & =0, \\
\phi_{3}^{\prime \prime}(1) \phi_{4}^{\prime \prime \prime}(1)-\phi_{3}^{\prime \prime \prime}(1) \phi_{4}^{\prime \prime}(1) & =0 .
\end{aligned}
$$

From (24), we can obtain the ratio of $W^{\prime}(0)$ and $W^{\prime \prime \prime}(0)$ as follows:

$$
\frac{W^{\prime \prime \prime}(0)}{W^{\prime}(0)}=-\frac{\phi_{2}(1)}{\phi_{4}(1)} .
$$

Utilizing (16) and (26), one can obtain the mode function equation of a pinned-pinned pipe conveying fluid as follows:

$$
W(\xi)=\phi_{2}(\xi)-\frac{\phi_{2}(1)}{\phi_{4}(1)} \phi_{4}(\xi) .
$$

Similarly, the mode function equations of a clamped-clamped pipe conveying fluid and a cantilevered pipe conveying fluid can be obtained as follows:

$$
\begin{aligned}
& W(\xi)=\phi_{3}(\xi)-\frac{\phi_{3}(1)}{\phi_{4}(1)} \phi_{4}(\xi), \\
& W(\xi)=\phi_{3}(\xi)-\frac{\phi_{3}^{\prime \prime}(1)}{\phi_{4}^{\prime \prime}(1)} \phi_{4}(\xi) .
\end{aligned}
$$



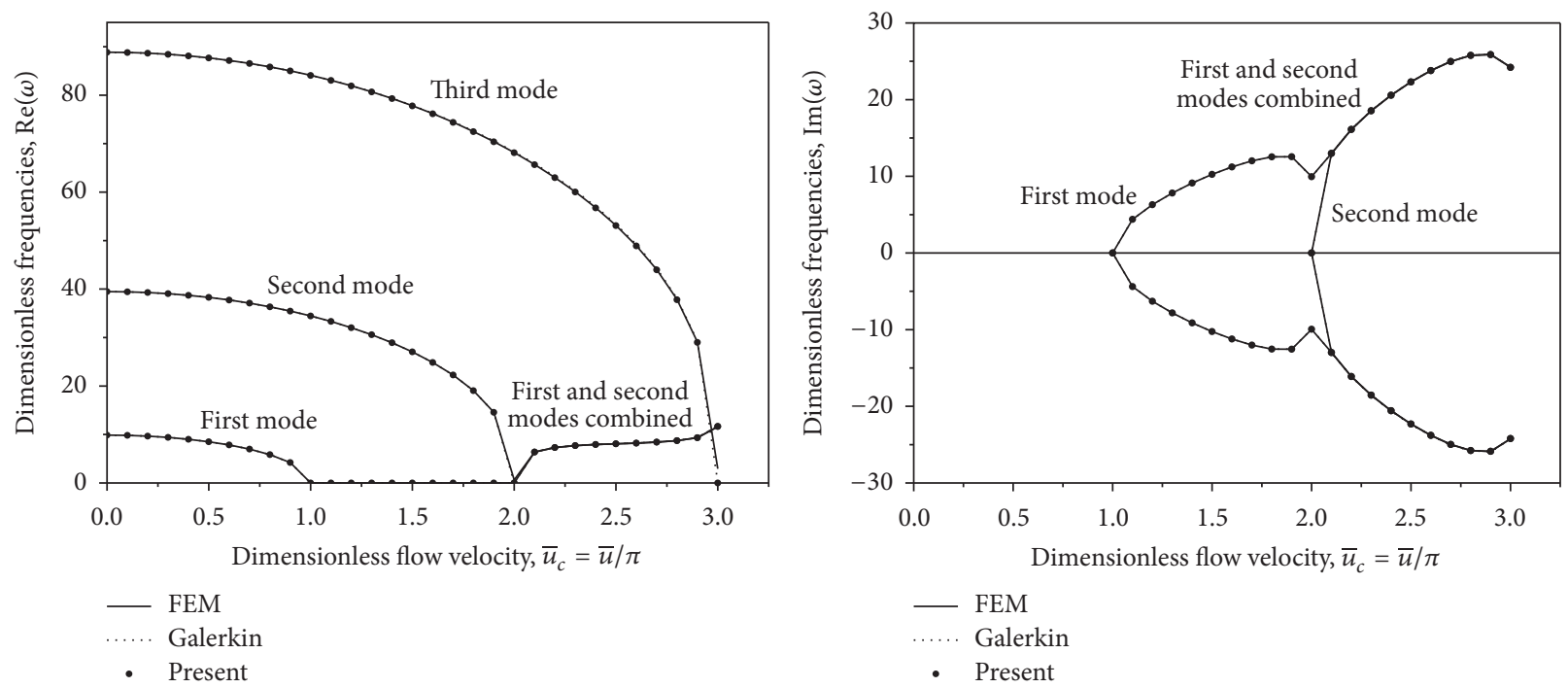

FIGURE 1: The real and imaginary components of the dimensionless frequencies of a pinned-pinned pipe conveying fluid as functions of the dimensionless flow velocity for $\beta=0.2$ and $c=0$.

\section{Numerical Results and Discussion}

4.1. The Stability of Pipe. The natural frequencies of pipe conveying fluid can be obtained from (25a) to (25c). It is impossible to obtain solutions of $(25 a)-(25 c)$ by direct methods because the roots of the problem cannot be expressed in simple explicit form in terms of $\omega$. So a trial and error procedure is adopted to seek the roots of (25a)-(25c). In this case, keeping $\beta$ and $c$ fixed for each set of calculations, the calculation of the real and imaginary part of the complex circular frequency $\omega$ is required for a series of values of $\bar{u}$. For most of the calculation, a computer program is used along the following lines [18]:

(i) Starting with $\bar{u}=0.1 \pi$ and trial values of $\operatorname{Re}(\omega)$ and $\operatorname{Im}(\omega)$ corresponding to $\bar{u}=0$, a minimizing procedure finds the appropriate values of $\operatorname{Re}(\omega)$ and $\operatorname{Im}(\omega)$ which meet $(25 a)-(25 c)$.

(ii) Taking an increment of $0.1 \pi$ in $\bar{u}$ and using the values of $\operatorname{Re}(\omega)$ and $\operatorname{Im}(\omega)$ found above as first approximations, the minimizing procedure finds the complex frequency for $\bar{u}=0.2 \pi$.

Thus the computer works continuously and the values of $\operatorname{Re}(\omega)$ and $\operatorname{Im}(\omega)$ are obtained for increasing values of $\bar{u}$, for any mode function of the system and any given $\beta$ and $c$. Some results are shown in Figures 1-3, in which the natural frequencies correspond to the three cases of support conditions specified in (21)-(23), respectively.

Figure 1 shows the real and imaginary components of the dimensionless frequencies of the lowest three modes of a pinned-pinned pipe as functions of the dimensionless flow velocity. Based on the calculated results of Galerkin method and the present method, the first mode frequency vanishes altogether at $\bar{u}=\pi$, indicating the onset of first mode divergence; beyond the critical $\bar{u}=\pi$, the first mode frequency becomes wholly imaginary, indicating the system buckles in the first mode; if the flow velocity is increased further, the second mode frequency vanishes altogether at $\bar{u}=2 \pi$, indicating the onset of second mode divergence; beyond the critical of $\bar{u}=2 \pi$, the frequencies of the first and second modes coalesce and frequencies become complex conjugates, indicating the system loses stability by couplemode flutter. Based on the calculated results of FEM, the frequency as function of the flow velocity is the same to the calculated results of other two methods except $\bar{u}=\pi$ and $\bar{u}=2 \pi$; the real component of the first frequency is 0.0246 at $\bar{u}=\pi$; the real component of the second frequency is 0.6326 at $\bar{u}=2 \pi$. So the FEM has a certain error at some flow velocities, such as $\bar{u}=\pi$ and $\bar{u}=2 \pi$, in comparison with Galerkin method and the present method.

Figure 2 shows that the real and imaginary components of the dimensionless frequencies of the lowest three modes of a clamped-clamped pipe as functions of the dimensionless flow velocity. Based on the calculated results of the present method, the first mode frequency vanishes altogether at $\bar{u}=$ $2 \pi$, indicating the onset of first mode divergence; beyond the critical $\bar{u}=2 \pi$, the first mode frequency becomes wholly imaginary, indicating the system buckles in the first mode; if the flow velocity is increased further, the system loses stability by couple-mode flutter in the first mode and second mode at $\bar{u}=2.9 \pi$. The calculated results of the three methods are different at $\bar{u}=2 \pi$; the real components of the first frequency are 0.9994 and 0.2111, respectively, with Galerkin method truncating four modes and FEM. According to Paidoussis and Sundararajan [3], the first mode frequency vanishes altogether. Therefore, Galerkin method and FEM have a certain error at some flow velocities, such as $\bar{u}=2 \pi$. As the flow velocity increases, the difference is more and more, between the calculated results of Galerkin method and the calculated results of other methods. The reason is that the number of the modes truncated is insufficient, according to the literature [19]. In this paper, the lower four modes 

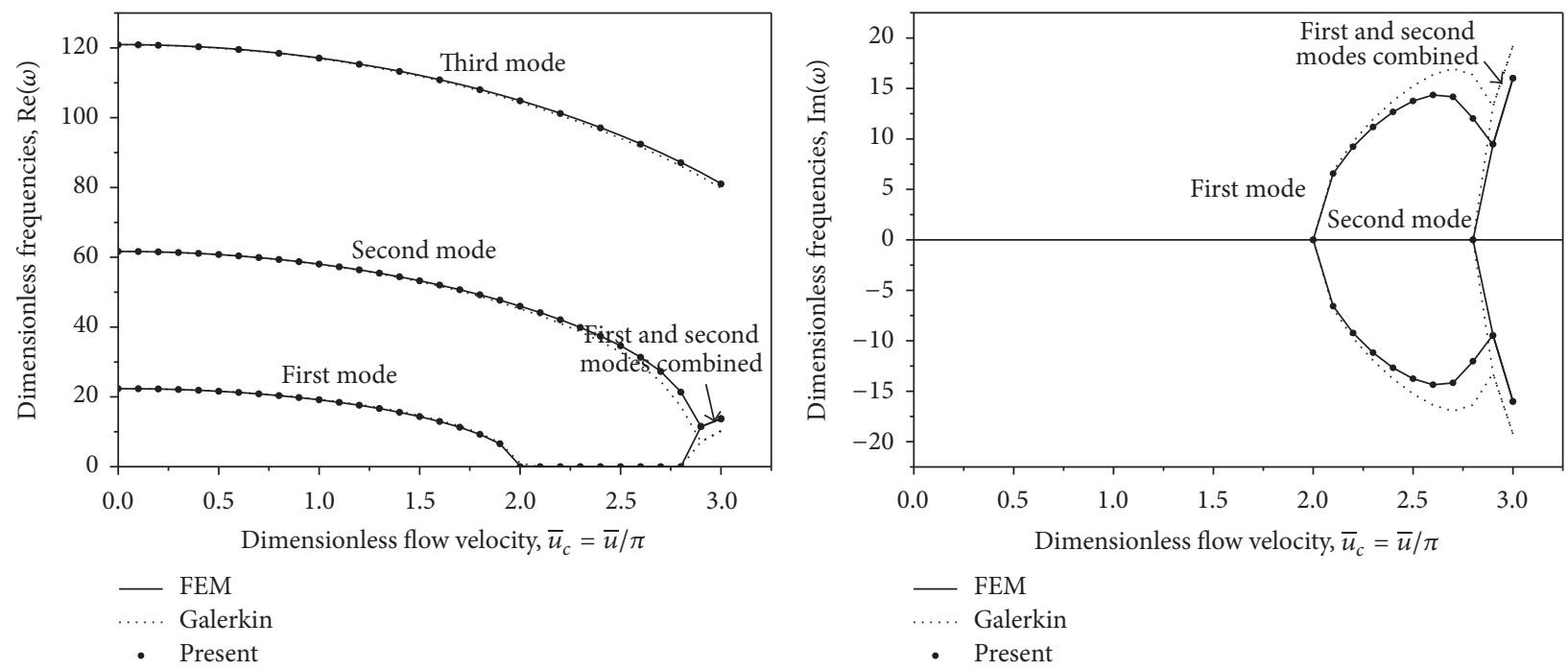

FIGURE 2: The real and imaginary components of the dimensionless frequencies of a clamped-clamped pipe conveying fluid as functions of the dimensionless flow velocity for $\beta=0.2$ and $c=0$.
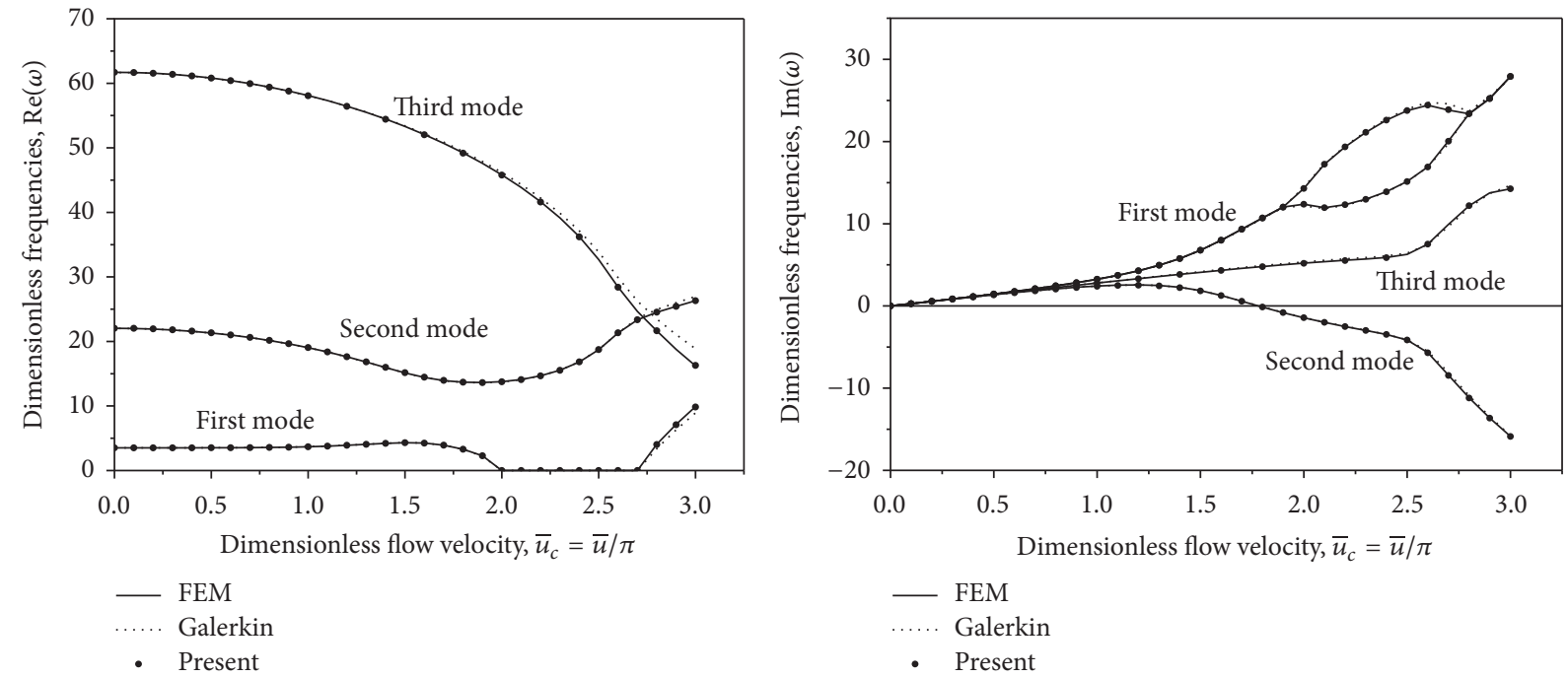

Figure 3: The real and imaginary components of the dimensionless frequencies of a clamped-free pipe conveying fluid as functions of the dimensionless flow velocity for $\beta=0.2$ and $c=0$.

are used in Galerkin method to solve the equation of small motion of pipes conveying fluid. The first and second modes of the clamped-clamped pipe conveying fluid represent the fifth and the higher modes of a clamped-clamped beam, when dimensionless flow velocity is relatively larger. So, the calculated results of Galerkin method are the difference from those of the other two methods.

Figure 3 shows the real and imaginary components of the dimensionless frequencies of the lowest three modes of a clamped-free pipe as functions of the dimensionless flow velocity. Based on the calculated results, beyond $\bar{u}=1.8 \pi$, the imaginary part of the second frequency becomes negative, and the real part of the second frequency is still positive, indicating the system flutters in the second mode through a Hopf bifurcation. Galerkin method has a certain error in comparison with FEM and the present, since the number of the modes truncated is insufficient.

4.2. The Mode Function of Pipe. From (27a) to (27c), one can find that the mode function of the pipe system is the function of $\bar{u}, \beta, c$, and $\omega$. These variables are not independent because they have an implicit relationship as (25a)-(25c). So, in generally, it is impossible to obtain the mode functions.

When flow velocity $\bar{u}$ is zero, (3) can be degenerated into the free vibration equation of tubular beam. For the free vibration of tubular beam, the natural frequencies are wholly real, and the vibration mode functions can be obtained in analytical form. The vibration mode functions of tubular 

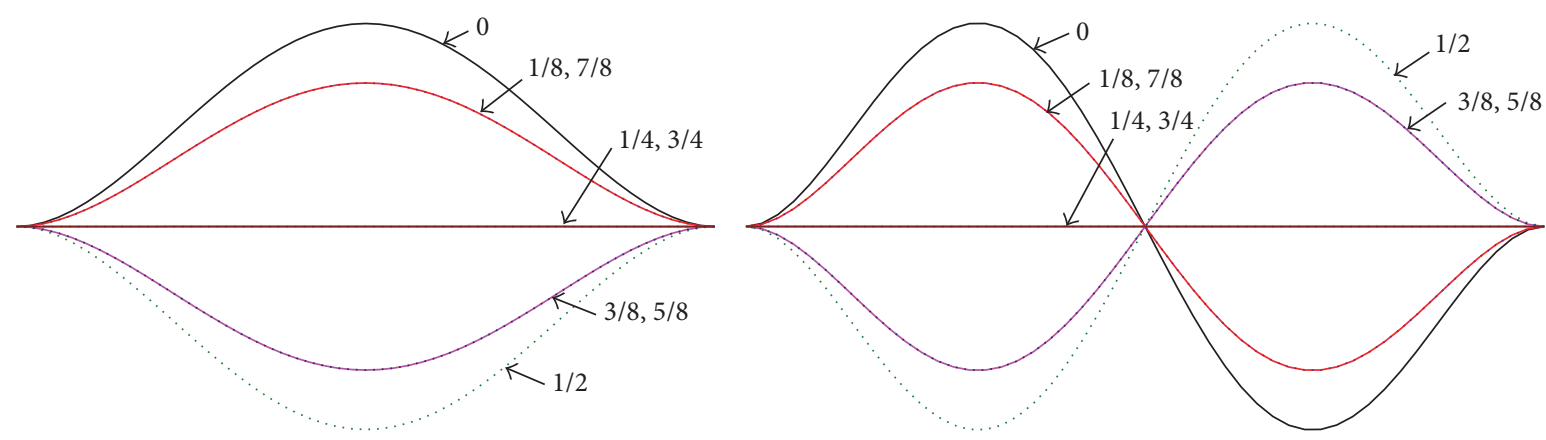

(a) $\bar{u}=0$
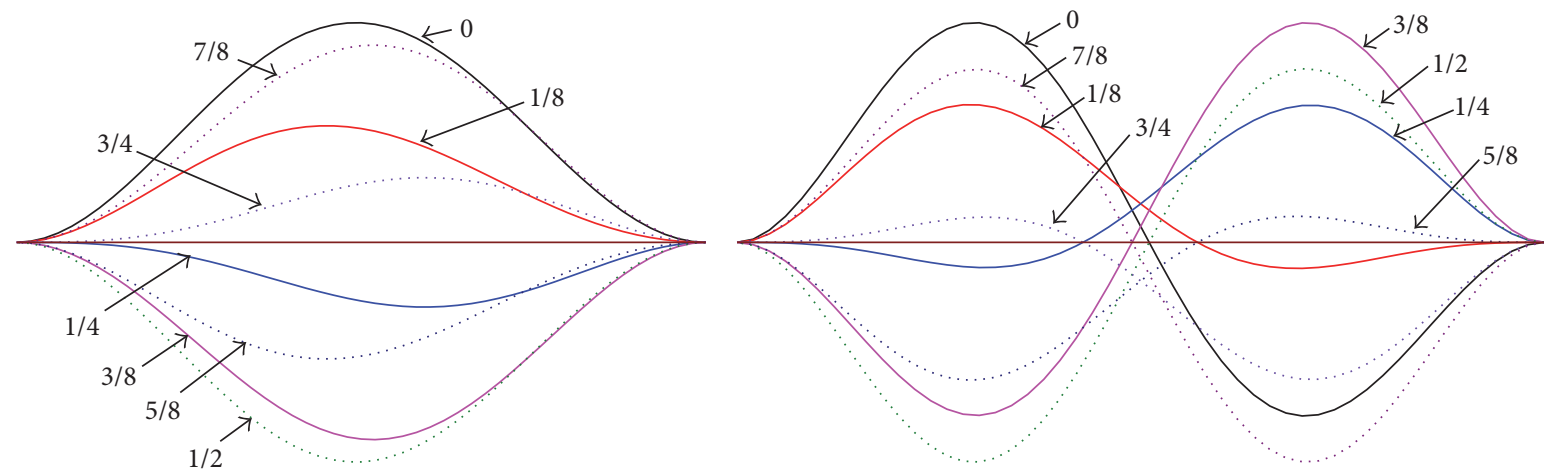

(b) $\bar{u}=1.5 \pi$

FIgURE 4: Variation of modal forms of the first two modes of a clamped-clamped pipe during a period of oscillation.

beam with several typical boundary conditions are obtained at $c=0$ as follows:

(i) A pinned-pinned tubular beam

$$
W(\xi)=\sin \left(\sqrt{\omega_{i}} \xi\right) .
$$

(ii) A clamped-clamped tubular beam

$W(\xi)$

$$
\begin{aligned}
= & \operatorname{ch}\left(\sqrt{\omega_{i}} \xi\right)-\cos \left(\sqrt{\omega_{i}} \xi\right) \\
& -\frac{\operatorname{ch} \sqrt{\omega_{i}}-\cos \sqrt{\omega_{i}}}{\operatorname{sh} \sqrt{\omega_{i}}-\sin \sqrt{\omega_{i}}}\left[\operatorname{sh}\left(\sqrt{\omega_{i}} \xi\right)-\sin \left(\sqrt{\omega_{i}} \xi\right)\right] .
\end{aligned}
$$

(iii) A clamped-free tubular beam

$W(\xi)$

$$
\begin{aligned}
= & \operatorname{ch}\left(\sqrt{\omega_{i}} \xi\right)-\cos \left(\sqrt{\omega_{i}} \xi\right) \\
& -\frac{\operatorname{sh} \sqrt{\omega_{i}}-\sin \sqrt{\omega_{i}}}{\operatorname{ch} \sqrt{\omega_{i}}+\cos \sqrt{\omega_{i}}}\left[\operatorname{sh}\left(\sqrt{\omega_{i}} \xi\right)-\sin \left(\sqrt{\omega_{i}} \xi\right)\right],
\end{aligned}
$$

where $i=1,2,3, \ldots$, corresponding to the various mode functions of the system. These results agree with literatures $[20,21]$.
The modal displacement patterns of pipe conveying fluid can be obtained with (27a)-(27c), and the modal displacement patterns of a clamped-clamped pipe and a clampedfree pipe can be seen in Figures 4 and 5, respectively. When flow velocity $\bar{u}$ is zero, the modal displacement patterns of pipe are classical normal modes, as seen in Figures 4(a) and 5(a). When $\bar{u}$ is not zero, the equation of motion of pipe contains the Coriolis term, which renders classical normal modes impossible, as seen in Figures 4(b) and 5(b). The motions associated with the symmetric modes of a clampedclamped pipe will produce the Coriolis force, which will induce the motion of antisymmetric modes. The Coriolis force, produced by the motions associated with the modes of a clamped-free pipe, is still in the direction against the motion along the whole pipe. This will tend to the oscillations. In general, the effect of the Coriolis force is more and more as the flow velocity increased.

\section{Conclusions}

The paper presented a new differential transformation method to study the free vibration of the pipe conveying fluid with various boundary conditions. The mode function equations and the frequency equations of the pipe conveying fluid were obtained by the present method. The frequency equation of pipe conveying fluid is implicit form in terms of $\omega$. A trial and error computer program was written for the $\omega$ of the pipe conveying fluid, so the solving procedure is 


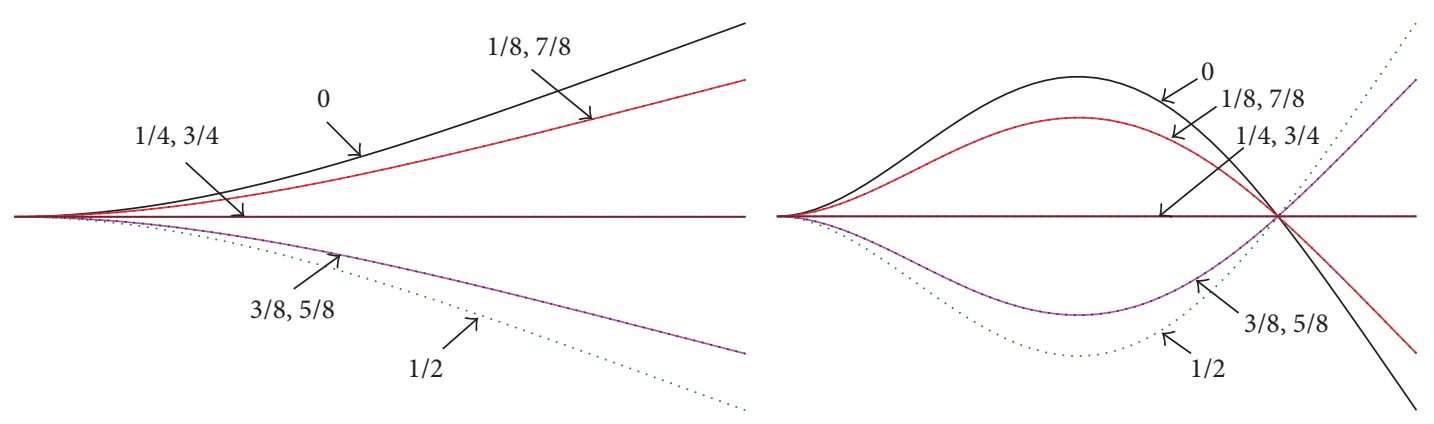

(a) $\bar{u}=0$

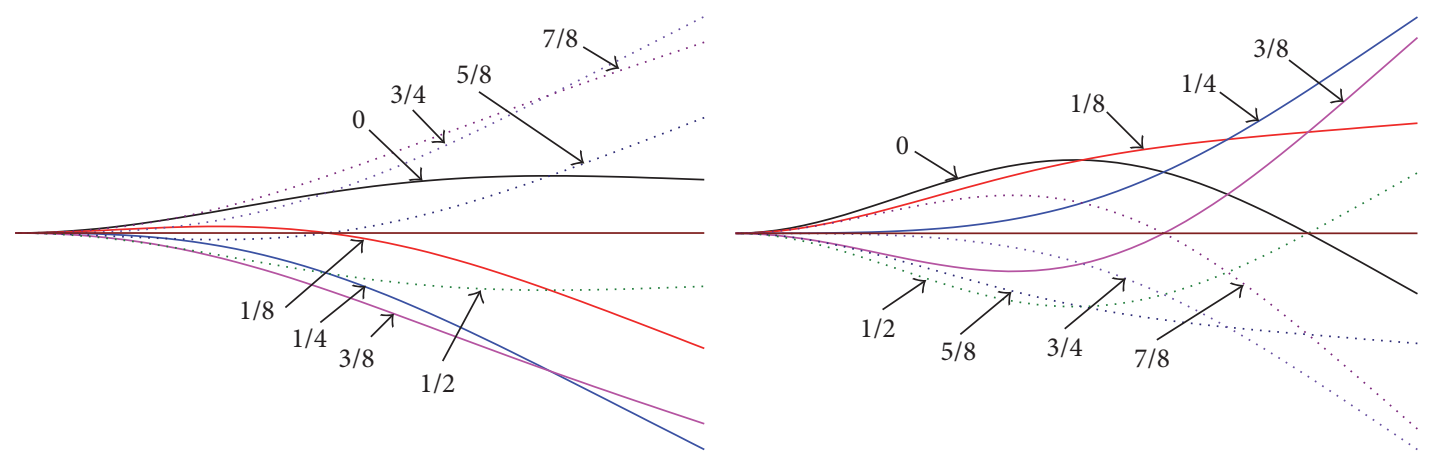

(b) $\bar{u}=1.5 \pi$

FIGURE 5: Variation of modal forms of the first two modes of a clamped-free pipe during a period of oscillation.

simple and rapid. The vibration mode equation has an explicit form in terms of $\xi$, so the vibration mode function of the pipe conveying fluid will be calculated when the $\omega$ of pipe conveying fluid is known. By comparing with the calculated results of Galerkin method and FEM, this analytical method is valid for the problem of dynamics and stability of pipes conveying fluid.

\section{Competing Interests}

The authors declare that they have no competing interests.

\section{Acknowledgments}

This work is supported by the National Natural Science Foundation of China (Grant no. 11102170); the project of Science \& Technology Department of Sichuan Province, China (Grant no. 2015GZ0037); the Opening Project of Sichuan Province University Key Laboratory of Bridge NonDestruction Detecting and Engineering Computing, China (Grant no. 2016QZJ03).

\section{References}

[1] H. Ashley and G. Haviland, "Bending vibrations of a pipe line containing flowing fluid," Journal of Applied Mechanics, vol. 17, pp. 229-232, 1950.

[2] R. W. Gregory and M. P. Paidoussis, "Unstable oscillation of tubular cantilevers conveying fluid. I. theory," Proceedings of the Royal Society A: Mathematical, Physical and Engineering Sciences, vol. 293, no. 1435, pp. 512-527, 1966.
[3] M. P. Paidoussis and C. Sundararajan, "Parametric and combination resonances of a pipe conveying pulsating fluid," Journal of Applied Mechanics, Transactions ASME, vol. 42, no. 4, pp. 780-784, 1975.

[4] M. J. Hannoyer and M. P. Paidoussis, "Instabilities of tubular beams simultaneously subjected to internal and external axial flows," Journal of Mechanical Design, Transaction of ASME, vol. 100, no. 2, pp. 328-336, 1978.

[5] K. S. Van, Dynamics and stability of curved pipes conveying fluid [M.S. thesis], MoGill University, 1986.

[6] A. K. Misra, M. P. Païdoussis, and K. S. Van, "On the dynamics of curved pipes transporting fluid. Part I: inextensible theory," Journal of Fluids and Structures, vol. 2, no. 3, pp. 221-244, 1988.

[7] L. G. Olson and D. Jamison, "Application of a general purpose finite element method to elastic pipes conveying fluid," Journal of Fluids and Structures, vol. 11, no. 2, pp. 207-222, 1997.

[8] G. L. Kuiper and A. V. Metrikine, "On stability of a clampedpinned pipe conveying fluid," Heron, vol. 49, no. 3, pp. 211-232, 2004.

[9] L. Wang and Q. Ni, “The nonlinear dynamic vibrations of a restrained pipe conveying fluid by Differential Quadrature Method," Journal of Dynamics and Control, vol. 2, no. 4, pp. 5661, 2004.

[10] Q. Qian, L. Wang, and Q. Ni, "Instability of simply supported pipes conveying fluid under thermal loads," Mechanics Research Communications, vol. 36, no. 3, pp. 413-417, 2009.

[11] F. Tornabene, A. Marzani, and E. I. Elishakoff, "Critical flow speeds of pipes conveying fluid using the generalized differential quadrature method," Advances in Theoretical and Applied Mechanics, vol. 3, pp. 121-138, 2010.

[12] Q. Ni, Z. L. Zhang, and L. Wang, "Application of the differential transformation method to vibration analysis of pipes conveying 
fluid," Applied Mathematics and Computation, vol. 217, no. 16, pp. 7028-7038, 2011.

[13] B. G. Sinir and D. D. Demir, "The analysis of nonlinear vibrations of a pipe conveying an ideal fluid," European Journal of Mechanics. B. Fluids, vol. 52, pp. 38-44, 2015.

[14] X. Y. Li, X. Zhao, and Y. H. Li, "Green's functions of the forced vibration of Timoshenko beams with damping effect," Journal of Sound and Vibration, vol. 333, no. 6, pp. 1781-1795, 2014.

[15] Y.-D. Li and Y.-R. Yang, "Forced vibration of pipe conveying fluid by the Green function method," Archive of Applied Mechanics, vol. 84, no. 12, pp. 1811-1823, 2014.

[16] F. J. Bourrieres, Sur un Phenomene d'Oscillations Auto-Entrenue en Mecanique des Fluides Reels, vol. 147 of Publications Scientifiques et Techniques du Ministere de l'Air, 1939.

[17] V. A. Ditkin and A. P. Prudnikov, Fromulaire pour le calcul Operationnel, Traduction du Russe par G. Carvallo, Masson \& Cie, Paris, France, 1967.

[18] R. W. Gregory and M. P. Paidoussis, "Unstable oscillation of tubular cantilevers conveying fluid. I. Theory," Proceedings of the Royal Society A: Mathematical, Physical and Engineering Sciences, vol. 293, no. 1435, pp. 512-527, 1966.

[19] H.-H. Qi and J. Xu, "Effect of Galerkin modal truncation on natural frequency analysis of a cantilevered pipe conveying fluid," Journal of Vibration and Shock, vol. 30, no. 1, pp. 148-151, 2011.

[20] R. E. Bishop and D. C. Johnson, The Mechanics of Vibration, Cambridge University Press, New York, NY, USA, 1960.

[21] M. P. Paidoussis, Fluid-Structure Interactions: Slender Structures and Axial Flow, vol. 1, Academic Press, California, Calif, USA, 1998. 


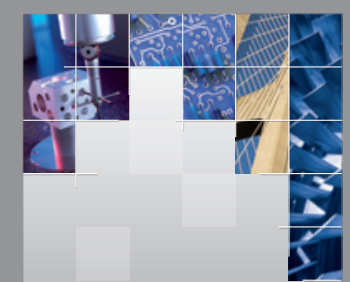

\section{Enfincering}
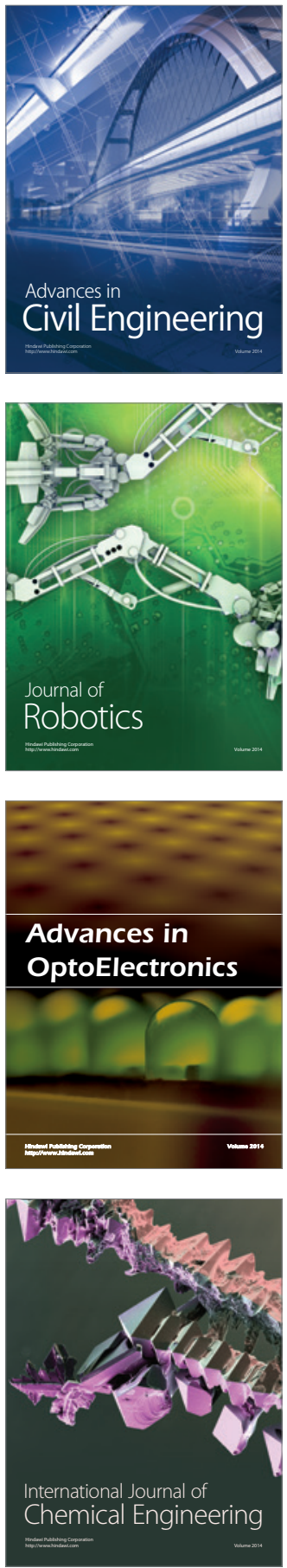

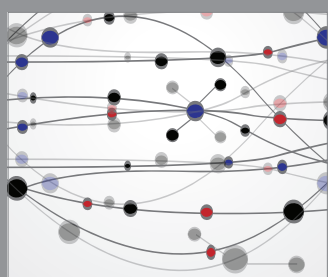

The Scientific World Journal

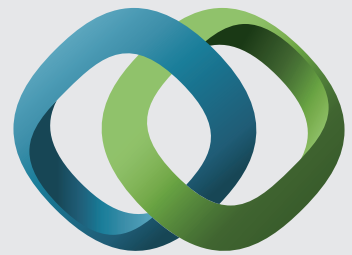

\section{Hindawi}

Submit your manuscripts at

https://www.hindawi.com
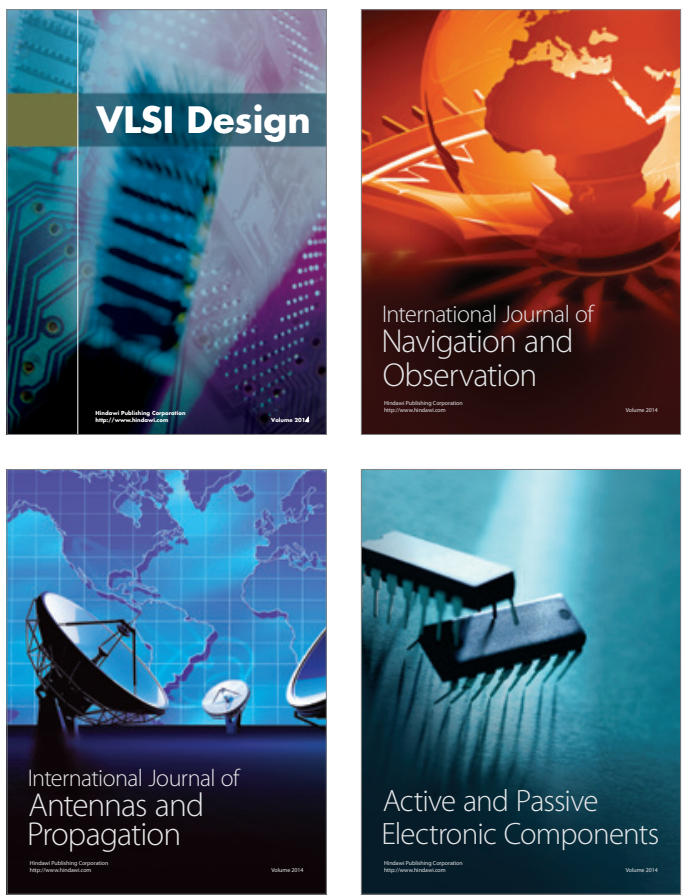
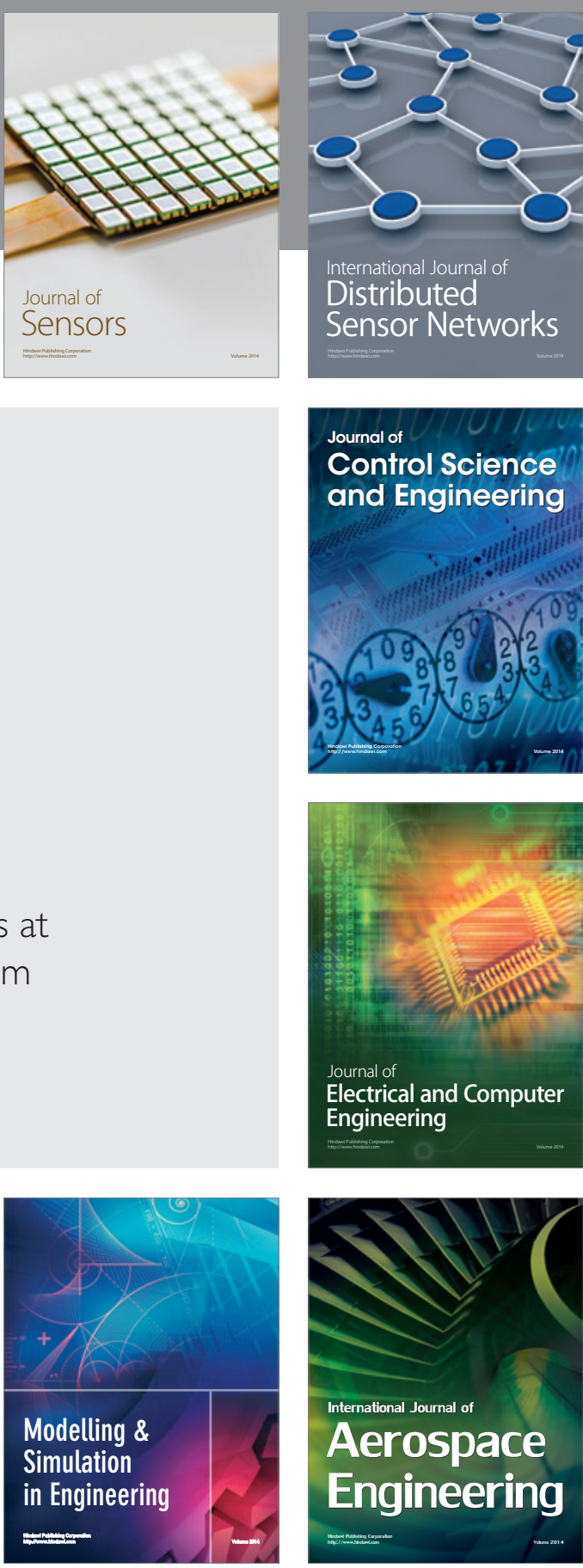

International Journal of

Distributed

Sensor Networks

$-$

Joumal of

Control Science

and Engineering
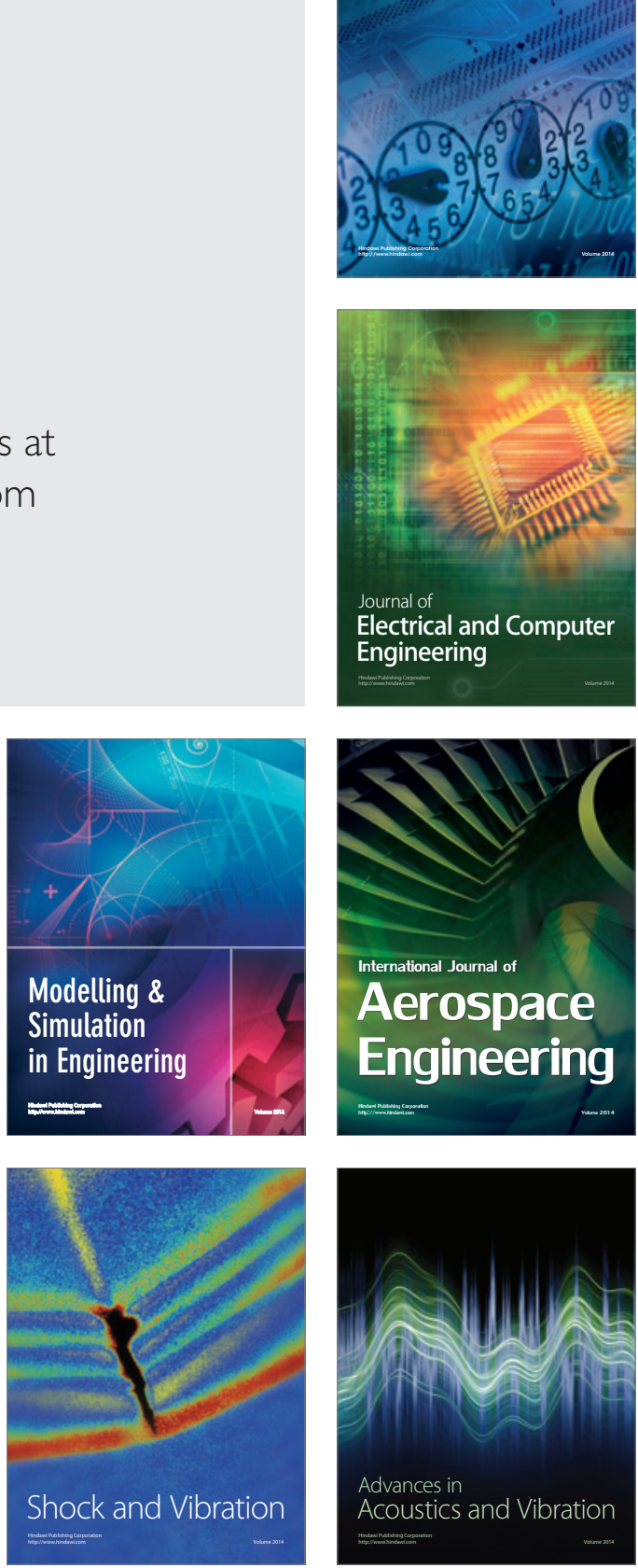